\title{
The Relation between Thermodynamics and Gravitational Constant $(G)$
}

\author{
Abed El Karim S. Abou Layla \\ Gaza City, Palestine \\ Email: a.k.aboulayla@gmail.com
}

How to cite this paper: Abou Layla, A. K. (2020) The Relation between Thermodynamics and Gravitational Constant $(G)$. Journal of High Energy Physics, Gravitation and Cosmology, 6, 680-691.

https://doi.org/10.4236/jhepgc.2020.64047

Received: March 16, 2020

Accepted: October 9, 2020

Published: October 12, 2020

Copyright (อ 2020 by author(s) and Scientific Research Publishing Inc. This work is licensed under the Creative Commons Attribution International License (CC BY 4.0).

http://creativecommons.org/licenses/by/4.0/

(c) (i) Open Access

\begin{abstract}
Although many centuries have elapsed since Newton set forth his gravitational law, physics has been unable so far to create an exact theoretical value for the universal gravitational constant $(G)$. Through a simple thought experience (i.e. it may not be possible to perform it), it can be concluded a mathematical formula which links three different physical sciences with each other: mechanics, electromagnetism and thermodynamics in a simple form, it is possible to find an exact value for the gravitational constant using this form. In fact, the importance of this research is that it also tells us more information about the electromagnetic and gravitomagnetic origin of masses, the negative and positive masses (i.e. matter and dark matter), and the smallest possible distance in the universe, which equals $1.0252 \times 10^{-56} \mathrm{~m}$.
\end{abstract}

\section{Keywords}

Thermodynamics, Gravitational Constant, Electromagnetic Mass, Radiation Mass, Khromatic Theory

\section{Introduction}

My first paper about the concept of "Precise Ideal Value of the Universal Gravitational Constant $G$ ' was introduced in 2017 [1], I have created a new law in physics without mathematical proof, through this law we can obtain a precise ideal value of $G$, and we find it equivalent to $6.674010551359 \times 10^{-11} \mathrm{~m}^{3} \cdot \mathrm{kg}^{-1} \cdot \mathrm{s}^{-2}$, and a relativistic value of $G$ which equals $6.674365970388 \times 10^{-11} \mathrm{~m}^{3} \cdot \mathrm{kg}^{-1} \cdot \mathrm{s}^{-2}$.

In this paper we will proof our new law of Gravitational Constant through a simple thought experience in which the electromagnetic force is equivalent with the gravitational force, to do this we will introduce a concept close to the electromagnetic mass that is the "radiation mass", which is considered as a dynamical explanation of inertial mass, relativistic and gravitational mass, knowing that 
there are two types of radiation mass, which are electromagnetic radiation mass $m^{r}$ and gravitomagnetic radiation mass $m^{r \prime}$.

Through the discussion, we calculated an exact value for the gravitational constant $G^{\prime}$, it is possible to compare the value we had got by theoretical means with the documented experimental values in CODATA gravitational tables and we will find that the values are close in both cases as shown in my first paper.

Next, we will look at the relationship between Gravitational Constant G and quantum mechanics, which takes the form, $G=\frac{1}{3} g_{e} \times 10^{-10}=6.674398 \times 10^{-11}$, where $g_{e}$ is the electron g-Factor which equals 2.00231930436256.

\section{Basic Notions}

\subsection{The Electromagnetic Field Notions}

In the case of an orbital electric charge $q$ has been under the influence of the central electric charge field $Q$ as in the classical atomic model [2].

Two harmonic magnetic poles $\boldsymbol{q}_{m}$ and $\boldsymbol{Q}_{m}$, are generated around each other as if they represent a magnetic atomic model accompanying the electric atomic model as shown in Figure 1.

By assuming that $\boldsymbol{r}_{1}, \boldsymbol{r}_{n}, \boldsymbol{r}_{\perp}$ are orthogonal unit vectors representing right-handed system, the values of orbital and central magnetic pole can be calculated as follows:

$$
\begin{aligned}
& \boldsymbol{q}_{m}=q \boldsymbol{v}_{\alpha}=q\left(v_{\alpha} \boldsymbol{r}_{n}\right) \\
& \boldsymbol{Q}_{m}=Q V_{\alpha} \boldsymbol{r}_{n}=Q \boldsymbol{v}_{\alpha}
\end{aligned}
$$

where

$\boldsymbol{q}_{m}$ : The orbital harmonic magnetic pole.

$Q_{m}$ : The central magnetic pole.

As a result of this new situation, magnetic forces are generated between these two harmonic poles, in addition to the emergence of new magnetic potential energy among them.

Consequently, the magnitude of these forces and potential energies will work with each other in the system, affecting the movement of the orbital and central charge.

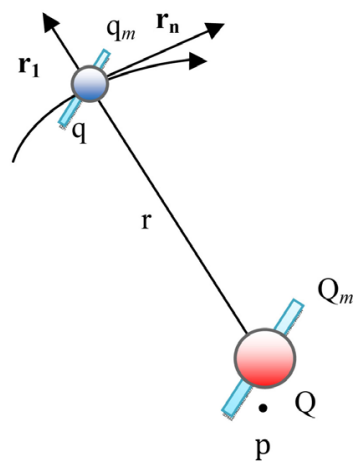

Figure 1. Orbital pole $\boldsymbol{q}_{m}$ moving under the central pole $Q_{m}$. 
Meaning that the classical atomic model actually became two overlapping atomic models that exchange interaction with each other in the system and affect the movement of objects in it according to the following equations:

$$
\begin{gathered}
\sum \boldsymbol{F}=\boldsymbol{F}_{e m}=\boldsymbol{F}_{e}+\boldsymbol{F}_{m} \\
\sum \varepsilon=\varepsilon_{k}+\varepsilon_{e m}=\varepsilon_{k}+\varepsilon_{e}+\varepsilon_{m} \\
\sum \boldsymbol{P}=P_{k}+P_{e m}
\end{gathered}
$$

where

$\sum F:$ Total forces affecting moving charge $q$.

$F_{e}$ : The electrical forces, $F_{e}=K \frac{q \times Q}{r^{2}}$.

$\boldsymbol{F}_{m}$ : The magnetic forces, $F_{m}=k_{m} \frac{\boldsymbol{q}_{m} \cdot \boldsymbol{Q}_{m}}{r^{2}}$.

$\sum \varepsilon$ : The total energy of charge.

$\varepsilon_{e m}:$ The total electromagnetic potential energy.

$\varepsilon_{e}:$ Electric potential energy, $\varepsilon_{e}=K \frac{q \times Q}{r}$.

$\varepsilon_{m}$ : The magnetic potential energy, $\varepsilon_{m}=k_{m} \frac{q_{m} \cdot Q_{m}}{r}$.

$P$. Total momentum of charge.

$\boldsymbol{P}_{k}$ : The kineticmomentum, $\boldsymbol{P}_{k}=m \boldsymbol{v}$.

$\boldsymbol{P}_{\text {em: }}$ electromagnetic momentum, $\boldsymbol{P}_{e m}=q \boldsymbol{A}$.

$A$ : The magnetic vector potential, $A=k_{m} \frac{Q_{m}}{r}$.

$k_{m}$ : coulomb magnetic constant, $k_{m}=\frac{k}{c^{2}}$.

\subsection{The Gravitomagnetic Field Notions}

There is a match in shape between equations of the atomic and the astronomical models [3], the difference is in the constants of these fields, so by letting,

$$
q \rightarrow m: Q \rightarrow M: k \rightarrow G
$$

we obtain the Gravitomagnetic field laws.

For example, we conclude from this there is gravitomagnetic forces between two masses according to the following equation:

$$
\boldsymbol{F}_{g \hat{m}}=\boldsymbol{F}_{g}+\boldsymbol{F}_{\hat{m}}
$$

where

$\boldsymbol{F}_{g}$ : is the gravitational force, $\boldsymbol{F}_{g}=G \frac{m M}{r^{2}}$.

$\boldsymbol{F}_{\hat{m}}:$ is the magnetic force, $\boldsymbol{F}_{\hat{m}}=G_{m} \frac{\boldsymbol{m}_{m} \cdot \boldsymbol{M}_{m}}{r^{2}}$.

$m, M$ : are the two masses.

$\boldsymbol{m}_{m}, \boldsymbol{M}_{m}$ : are the two magnetic poles, $\boldsymbol{m}_{m}=m \boldsymbol{v}, \boldsymbol{M}_{m}=M \boldsymbol{v}$.

$r$ is the distance between masses. 
$G$ : is the gravitational constant.

$G_{m}$ : is the gravitational magnetic constant, $G_{m}=\frac{G}{c^{2}}$.

Gravitational and magnetic force equations can be reformulated as follows:

$$
\begin{gathered}
F_{g}=\frac{G_{m}}{c^{2}} \frac{\varepsilon \mathcal{E}}{r^{2}} \\
F_{\hat{m}}=G_{m} \frac{\boldsymbol{p} \cdot \boldsymbol{P}}{r^{2}}
\end{gathered}
$$

where

$\varepsilon, \mathcal{E}:$ are the two energy of masses, $\varepsilon=m c^{2}, \mathcal{E}=M c^{2}$.

$\boldsymbol{p}, \boldsymbol{P}:$ are the two momentum of masses, $\boldsymbol{p}=m \boldsymbol{v}, \boldsymbol{P}=M \boldsymbol{v}$.

\section{Charges as Origin of Mass}

For each orbital charge $q$ moving under the influence of central charge $Q$, acquires an additional quantity of electromagnetic momentum $P_{e m}$ [4].

As a result of this new situation, an additional mass is generated that can be known as an electromagnetic radiation mass $m^{r}$, so it can be concluded that the moving electrical charge is a massless charge $q$ that contains an radiation mass $m^{r}$ that is considered as the nucleus of this charge, as it is clarified in Figure 2.

In short, the moving electric charge $q$ is a mechanically kahromatic charge $q_{k}$.

\subsection{The Concept of Electromagnetic Radiation Mass}

We can prove that the electromagnetic radiation mass $m^{r}$ is a mass which is equivalent to the potential energy between two electric charges $\mathcal{E}_{e}$ according to the following equation:

$$
\varepsilon_{e}=m^{r} c^{2}
$$

where:

$$
\varepsilon_{e}=k \frac{q Q}{r}
$$

we can find the radiation mass $m^{r(x)}$ for any distance $r_{x}$ as the following,

$$
m^{r(x)}=\frac{\varepsilon_{e}}{c^{2}}=\frac{1}{c^{2}}\left(k \frac{q Q}{r_{x}}\right)=\frac{k}{c^{2}} \frac{q Q}{r_{x}}=k_{m} \frac{q Q}{r_{x}}
$$

the radiation mass will be negative if the electric charges have opposite signs and positive if the electric charges have like-signed.

\subsection{Radiation Momentum $P r$}

The electromagnetic momentum equation takes the following form

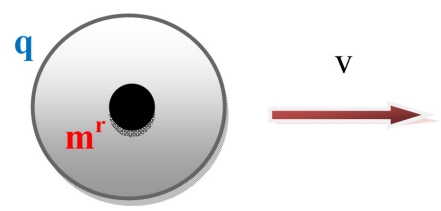

Figure 2. Kahromatic charge. 


$$
\boldsymbol{P}_{e m}=q \boldsymbol{A}
$$

Then we get

$$
\boldsymbol{P}_{e m}=q \boldsymbol{A}=q\left(k_{m} \frac{\boldsymbol{Q}_{m}}{r}\right)=k_{m} \frac{q\left(Q \boldsymbol{V}_{\alpha}\right)}{r}=\left(k_{m} \frac{q Q}{r}\right) \boldsymbol{V}_{\alpha}=m^{r} \boldsymbol{V}_{\alpha}=\boldsymbol{p}^{r}
$$

The radiation momentum can be writing on the following form

$$
\boldsymbol{p}^{r}=m^{r} \boldsymbol{V}_{\alpha}
$$

where:

$P$ : radiation momentum equals the magnetic potential momentum $\boldsymbol{P}_{e m}$.

$m^{r}$ : The radiation mass.

$A$ : The magnetic vector potential.

$Q_{m}$ : The central magnetic pole.

\subsection{The Radiation Force $\boldsymbol{F}_{r}$}

The forces of attraction or repulsion between two radiation masses can be calculated by law of universal gravitation

$$
F_{r}=-G \frac{m^{r} M^{r}}{r^{2}}
$$

where

$F_{r}:$ is the Radiation force, it is attractive if the radiation masses have like-signed (i.e., $F$ is negative) and repulsive if opposite signs (i.e., $F$ is positive)

$m^{r}, M^{r}$ : are the two radiation masses,

$r$ is the distance between masses,

$G$ : is the gravitational constant.

\section{The Kahromatic Particles}

The kahromatic particles contains from two single kahromatic charges which possible to be positive or negative charges, bound to each other by the Coulomb force and Radiation force in the case equivalent as shown in Figure 3.

\subsection{Velocity of Kahromatic Particle}

The kahromatic particle looks like a massless hydrogen atom, has constant, one direction velocity which equals the orbital speed of electron in Bohr's Hydrogen Atom.

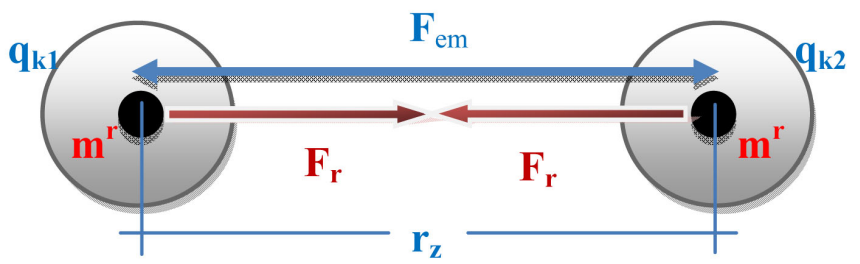

Figure 3. Two similar kahromatic charges $q_{k}$ in the case equivalent. 


\subsection{Condition of Mechanical Equilibrium}

For a particle to be in mechanical equilibrium, the net force must be zero, in the form of an equation, this condition is:

$$
F_{n e t}=0
$$

Then,

$$
\begin{gathered}
F_{e m}-F_{r}=0 \Rightarrow F_{r}=F_{e m} \\
G \frac{\left(m^{r(z)}\right)^{2}}{r_{z}^{2}}=k \frac{q^{2}}{r_{z}^{2}}
\end{gathered}
$$

Thus

$$
\begin{gathered}
\left(m^{r(z)}\right)^{2}=\frac{k}{G} q^{2}=\delta^{2} q^{2} \\
m^{r(z)}= \pm q \delta
\end{gathered}
$$

As it is the first condition of getting the kahromatic particles in mechanical equilibrium.

Where

$\delta:$ is the kahromatic equilibrium constant and its unit is a kilogram/colum.

$r_{z}$ : is defined as the critical distance between kahromatic charges.

$m^{r(z)}$ : is the critical radiation mass.

In this case, the kahromatic particles act as free-floating neutral particles whose collisions are subject to the laws and assumptions of the ideal gas theory.

\section{Thermodynamics as Origin of Three Sciences}

\subsection{The Relation between Kahromatic Gas and Thermodynamics}

Suppose that we have a piston contains within it kahromatic particles gas has constant, one direction motion (i.e., without spin or vibrations), which can be represented by the classical ideal gas, as shown in Figure 4 .

Assuming that $N$ is the number molecules of theparticles gas, and that the weight of the molecule of this gas is $m_{0}$, therefore the number of moles of this gas $n$ is given or expressed by the equation:

$$
n=\frac{m}{M}
$$

where

$$
m=m_{0} \times N, M=m_{0} \times N_{A}
$$

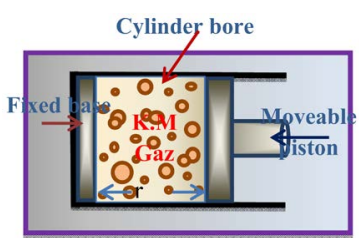

Figure 4. Sketch of piston. 
In this case, the general law of gases can be applied to the piston as the following:

$$
n R T_{z}=2 \varepsilon_{\text {total }}^{r}
$$

where

$n$ : is the number of gas molecules.

$\varepsilon_{\text {total }}^{r}$ : is the total kinetic energy of kahromatic gas.

$R$ : is the gas constant $\mathrm{J} \cdot \mathrm{K}^{-1} \cdot \mathrm{mol}^{-1}$.

$T_{z}$ : is the temperature of gas in equivalent case $\mathrm{K}$.

On the other hand, the total energy of gas $\varepsilon_{\text {total }}^{r}$ is given by the equation:

$$
\varepsilon_{\text {total }}^{r}=N \varepsilon^{r}
$$

where $\varepsilon^{r}$ is the radiation kinetic energy of one particle, which can be formulated as follows:

$$
\begin{aligned}
& \varepsilon^{r}=\left(\frac{1}{2} m^{r} v^{2}\right)=\frac{1}{2} \frac{\varepsilon_{e}}{c^{2}} v^{2}=\frac{1}{2} \alpha^{2} \varepsilon_{e} \\
&= \frac{1}{2} \alpha^{2} \frac{1}{r_{z}}\left(k \frac{q^{2}}{r_{z}}\right) r_{z}=\frac{1}{2} \alpha^{2}\left(k \frac{q^{2}}{r_{z}^{2}}\right) r_{z}=\frac{1}{2} \alpha^{2} F r_{z} \\
& 2 N \varepsilon^{r}=\alpha^{2} F N r_{z}
\end{aligned}
$$

Thus, the right side of Equation (5.1) can be replaced by the equivalent of the last equation, so that we have the following equation:

$$
n R T_{z}=\alpha^{2} F N r_{z}
$$

Therefore

$$
\begin{aligned}
& n K_{m} R T_{z}=\alpha^{2} K_{m} F \cdot N r_{z} \\
& \frac{T_{z}}{\alpha^{2} K_{m} F}=\frac{N r_{z}}{K_{m} n R}=\propto^{2}
\end{aligned}
$$

So, we can get the relationships

$$
\begin{aligned}
\frac{T_{z}}{\alpha^{2} K_{m} F} & =\propto^{2} \\
\frac{N r_{z}}{K_{m} n R} & =\propto^{2}
\end{aligned}
$$

where

$K_{m}$ : is the magnetic coulomb constant N/A ${ }^{2}$.

$\propto$ : is the proportionality value.

\subsubsection{The Critical Distance $r_{z}$ between Two Kahromatic Charges}

From (5.3) we get:

$$
\begin{gathered}
N r_{z}=\propto^{2} n K_{m} R=\propto^{2} \frac{m}{M} K_{m} R=\propto^{2} \frac{m}{m_{0}} K_{m} \frac{R}{N_{A}}=\propto^{2} \frac{m}{m_{0}} K_{m} K_{B} \\
m_{0} N r_{z}=m\left(\propto^{2} K_{m} K_{B}\right) \\
r_{z}=\propto^{2} K_{m} K_{B}
\end{gathered}
$$


As it is the equation of the critical distance between kahromatic charges. Where

$k_{B}$ : is the Boltzmann constant $\mathrm{J} / \mathrm{K}$.

The critical value of distance $r_{z}$ is a minimum of length, which equals:

$$
r_{z}=1.3806 \times 10^{-36}
$$

\subsubsection{The Equation of the Critical Distance in Gravitomagnetic Field} Using (2.1), we conclude that

$$
r_{z}^{\prime}=\propto^{2} G_{m} K_{B}
$$

The value of distance $r_{z}^{\prime}$ is the smallest possible distance in the universe, which equals:

$$
r_{z}^{\prime}=1.0252 \times 10^{-56}
$$

\subsection{The Relation between Temperature and Magnetic Vector Potential}

We know that:

$$
\begin{gathered}
\alpha^{2} K_{m} F=\alpha^{2} K_{m}\left(k \frac{q^{2}}{r^{2}}\right)=K_{m}\left(\frac{k}{c^{2}} \frac{q^{2}}{r^{2}} \alpha^{2} c^{2}\right) \\
\alpha^{2} K_{m} F=\left(K_{m} \frac{q v}{r}\right)^{2}=A^{2}
\end{gathered}
$$

where $A$ is the magnetic vector potential, and thus the Equation (5.4) can be reformulated as follows

$$
\frac{T_{z}}{\alpha^{2} K_{m} F}=\frac{T_{z}}{A^{2}}=\propto^{2}
$$

Therefore

$$
T_{z}=\propto^{2} \boldsymbol{A}^{2}
$$

Which means that the temperature of the system is directly proportional to the square of the magnetic vector potential within the system.

The last equation represents the relationship between only two different physical sciences: electromagnetism and thermodynamics.

\subsection{The Relation between Gravitational, Electromagnetic Theory, and Thermodynamics}

The equation of critical radiation mass has the following formula

$$
m^{r(z)}=K_{m} \frac{q^{2}}{r_{z}}
$$

Therefore,

$$
\begin{gathered}
m^{r(z)} r_{z}=K_{m} q^{2} \\
m^{r(z)}\left(\propto^{2} K_{m} K_{B}\right)=K_{m} q^{2}
\end{gathered}
$$




$$
\begin{gathered}
q^{2}=m^{r(z)} \propto^{2} K_{B} \\
q=\propto^{2}\left(\frac{m^{r(z)}}{q}\right) K_{B}
\end{gathered}
$$

As the system is within kahromatic equilibrium state, so

$$
q=\propto^{2} \delta K_{B}
$$

The last equation represents the relationship between three different physical sciences: gravitational, electromagnetism and thermodynamics, which explains that the Boltzmann constant is origin of electric charge.

\section{General Gravitational Constant}

There are many values of gravitational constant, including the exact and the relativistic value for gravitational constant.

\subsection{The Absolute Value for General Gravitational Constant}

The absolute value is a the exact value of the gravitational constant without relativity, which can be found as follows:

By squaring the two sides of the Equation (5.7), we get the following:

$$
q^{2}=\propto^{4} \delta^{2}\left(k_{B}\right)^{2}
$$

Then,

$$
\begin{aligned}
q^{2} & =\propto^{4} \frac{k}{G}\left(k_{B}\right)^{2} \\
G & =\propto^{4} k\left(\frac{k_{B}}{q}\right)^{2}
\end{aligned}
$$

The last equation can be reformulated in terms of the elementary charge e and speed of light $\mathrm{c}$ to have the following form:

$$
\propto^{4} k_{m}\left(\frac{k_{B}}{e}\right)^{2} c^{2}=10^{-19}\left(\frac{k_{B}}{e} c\right)^{2}=G
$$

In accordance with relationship

$$
G=10^{-19}\left(\frac{k_{B}}{e} c\right)^{2}
$$

And it is the exact formula for determining the value of the general gravitational constant, which known in the khromatic theory as "The Law Of Gravitational Constant".

Where:

G: absolute gravitational constant $\left(\mathrm{m}^{3} \cdot \mathrm{kg}^{-1} \cdot \mathrm{s}^{-2}\right)$.

$C$ : Speed of light in vacuum $(\mathrm{m} / \mathrm{s})$.

e: Elementary charge (colum).

$k_{B}$ : Boltzmann constant $(\mathrm{J} / \mathrm{K})$. 


\subsection{The Relativistic Value for General Gravitational Constant}

The relativistic value can be calculated as follows:

$$
F=G \frac{M m}{r^{2}}
$$

From the theory of relativity, we have

$$
m=\gamma m_{0}: M=\gamma M_{0} \text { as } \gamma=\frac{1}{\sqrt{1-\left(\frac{v}{c}\right)^{2}}}
$$

Then we get:

$$
F=\gamma^{2} G \frac{M_{0} m_{0}}{r^{2}}=G_{r} \frac{M_{0} m_{0}}{r^{2}}
$$

In last equation we assuming that,

$$
G_{r}=\gamma^{2} G
$$

where

$G_{r}$ : relativistic gravitational constant,

$v$. is the orbital speed of electron in an atom of hydrogen.

\section{Discussion}

In this discussion, we are going to find the proportionality constant $\propto$, and the gravitation constant $G$ as a numerical value.

After that, it is possible to compare the value of the gravitation constant $G$ we had got by theoretical means with the documented experimental values in CODATA gravitational tables, and we will show that the values are close in both cases [5].

\subsection{The Calculation of Proportionality Constant $\propto$}

We conclude from Equation (5.5) that proportionality value $\propto$ is a constant value, accordingly, for any the electron velocity $\mathrm{v}$, and $q$ is equal to the elementary charge, e, the constant value $\propto$ can be calculated as follows:

$$
\begin{gathered}
q=e \\
m^{r(z)}=\delta q=1.859249 \times 10^{-9} \mathrm{~kg} \\
r_{z}=K_{m} \frac{q^{2}}{m^{r(z)}}=K_{m} \frac{q}{\delta}=1.380648 \times 10^{-36} \mathrm{~m} \\
T_{z} K_{B}=\varepsilon^{r(z)}=m^{r(z)} v^{2}=1.85927 \times 10^{-5} \mathrm{~J} \\
\boldsymbol{A}=K_{m} \frac{q_{m}}{r_{z}}=K_{m} \frac{q v}{r_{z}}=1.160452 \times 10^{12} \text { tesla } \cdot \text { metre }
\end{gathered}
$$

where

$$
\begin{gathered}
v=100 \mathrm{~m} / \mathrm{s} \\
e=1.602176 \times 10^{-19} \mathrm{C}
\end{gathered}
$$




$$
\begin{gathered}
k_{B}=1.380648 \times 10^{-23} \mathrm{~J} / \mathrm{K} \\
k_{m}=10^{-7} \\
\delta=1.1604522 \times 10^{10} \mathrm{~kg} / \text { colum }
\end{gathered}
$$

Thus

$$
\begin{gathered}
T=\frac{m^{r(z)} v^{2}}{K_{B}}=1.346643 \times 10^{18} \\
A^{2}=1.346649 \times 10^{24} \\
\propto^{2}=\frac{T}{A^{2}}=\frac{1.346649 \times 10^{18}}{1.346649 \times 10^{24}}=10^{-6}
\end{gathered}
$$

Then we get

$$
\propto=10^{-3}
$$

\subsection{The Absolute Value for Gravitational Constant $G$}

Using substitution in the value of constants from an abbreviated list of the 2014 CODATA recommended values of the fundamental constants of physics and chemistry, we get the absolute value of the gravitation constant equaling:

C: $299792458(\mathrm{~m} / \mathrm{s})$.

e: $1.6021766208(98) \times 10^{-19}$ (colum).

$k_{B}: 1.38064852(79) \times 10^{-23}(\mathrm{~J} / \mathrm{K})$.

So,

$$
\begin{gathered}
G=10^{-19}\left(\frac{1.38064852 \times 10^{-23}}{1.6021766208 \times 10^{-19}} \times 299792458\right)^{2} \\
G=6.674010551359 \times 10^{-11} \mathrm{~m}^{3} \cdot \mathrm{kg}^{-1} \cdot \mathrm{s}^{-2}
\end{gathered}
$$

\subsection{The Relativistic Value for General Gravitational Constant}

From equation,

$$
G_{r}=\gamma^{2} G
$$

where

$$
\begin{gathered}
\gamma=\frac{1}{\sqrt{1-\alpha^{2}}} \\
\alpha \text { : fine-structure constant }=7.2973525664 \times 10^{-3}
\end{gathered}
$$

And on calculation the relativistic value of gravitational constant we get:

$$
G_{r}=6.674365970388 \times 10^{-11} \mathrm{~m}^{3} \cdot \mathrm{kg}^{-1} \cdot \mathrm{s}^{-2}
$$

\section{Acknowledgements}

I am fully thankful for the Palestinian President Mahmoud Abbas, for his wise management of the Corona crisis, as well as for his honorable national stances in international forums in defense of the Palestinian cause. 


\section{Conflicts of Interest}

The author declares no conflicts of interest regarding the publication of this paper.

\section{References}

[1] Abou Layla, A.K. (2017) Precise Ideal Value of the Universal Gravitational Constant G. Journal of High Energy Physics, Gravitation and Cosmology, 3, 248-253. https://doi.org/10.4236/jhepgc.2017.32020 https://www.gsjournal.net/Science-Journals/Research\%20Papers-Gravity/Download $\underline{16722}$

[2] Abou Layla, A.K. (2017) Calclation: The Exact Value of Gravitational Constant, LAP LAMBERT Academic Publishing, Dubai, 17.

https://www.amazon.com/dp/3330337257/ref=cm sw r fa dp U -DfZAb9WVV8 QK

[3] Abou Layla, A.K. (2017) Symmetry in Equations of Motion between the Atomic and Astronomical Models. Journal of High Energy Physics, Gravitation and Cosmology, 3, 328-338. https://doi.org/10.4236/jhepgc.2017.32028 http://www.scirp.org/journal/PaperDownload.aspx?paperID $=75700$

[4] Abou Layla, A.K. (2017) Calclation: The Exact Value of Gravitational Constant, LAP LAMBERT Academic Publishing, Dubai, 31,

https://www.amazon.com/dp/3330337257/ref=cm sw r_fa dp_U -DfZAb9WVV8 QK

[5] Abou Layla, A.K. (2017) Precise Ideal Value of the Universal Gravitational Constant G. Journal of High Energy Physics, Gravitation and Cosmology, 3, 251-252. https://doi.org/10.4236/jhepgc.2017.32020 https://www.gsjournal.net/Science-Journals/Research\%20Papers-Gravity/Download $\underline{16722}$ 